\title{
THE SYMPLECTICITY OF THE MAGNUS REPRESENTATION FOR HOMOLOGY COBORDISMS OF SURFACES
}

\author{
TAKUYA SAKASAI
}

The Magnus matrix is an algebraic invariant assigned to each homology cobordism of a surface. We show that this matrix satisfies an equality which can be regarded as a non-commutative symplectic relation.

\section{INTRODUCTION}

Let $\Sigma_{g, 1}$ be a compact connected oriented surface of genus $g \geqslant 1$ with one boundary component. We consider a triplet $\left(M, i_{+}, i_{-}\right)$consisting of a homology cobordism $M$ of $\Sigma_{g, 1}$ and two markings $i_{+}, i_{-}$of $\partial M$. More precisely, the compact oriented 3-manifold $M$ and the two embeddings $i_{+}, i_{-}: \Sigma_{g, 1} \rightarrow \partial M$ satisfy

(1) $i_{+}$is orientation-preserving and $i_{-}$is orientation-reversing,

(2) $\partial M=i_{+}\left(\Sigma_{g, 1}\right) \cup i_{-}\left(\Sigma_{g, 1}\right)$ and $i_{+}\left(\Sigma_{g, 1}\right) \cap i_{-}\left(\Sigma_{g, 1}\right)=i_{+}\left(\partial \Sigma_{g, 1}\right)=i_{-}\left(\partial \Sigma_{g, 1}\right)$,

(3) $\left.i_{+}\right|_{\partial \Sigma_{g, 1}}=\left.i_{-}\right|_{\partial \Sigma_{g, 1}}$,

(4) $i_{+}, i_{-}: H_{*}\left(\Sigma_{g, 1}\right) \rightarrow H_{*}(M)$ are isomorphisms.

We call such an object a homology cylinder (over $\Sigma_{g, 1}$ ), which has its origin in Habiro [4], Garoufalidis-Levine [3] and Levine [7]. Two homology cylinders are said to be isomorphic if there exists an orientation-preserving diffeomorphism between the underlying 3 -manifolds which is compatible with the markings. The set $\mathcal{C}_{g, 1}$ of all isomorphism classes of homology cylinders has a monoid structure defined by

$$
M \cdot N=\left(M \cup_{i_{-} \circ\left(j_{+}\right)^{-1}} N, i_{+}, j_{-}\right)
$$

for two homology cylinders $M=\left(M, i_{+}, i_{-}\right), N=\left(N, j_{+}, j_{-}\right)$. The unit of $\mathcal{C}_{g, 1}$ is $\left(\Sigma_{g, 1} \times I\right.$, id $\times 1$, id $\left.\times 0\right)$, where collars of $i_{+}\left(\Sigma_{g, 1}\right)$ and $i_{-}\left(\Sigma_{g, 1}\right)$ are stretched halfway along $\left(\partial \Sigma_{g, 1}\right) \times I$. Typical examples of homology cylinders come from the mapping class group $\mathcal{M}_{g, 1}$ of $\Sigma_{g, 1} ;$ that is the group of isotopy classes of diffeomorphisms of $\Sigma_{g, 1}$ which restrict to the identity on $\partial \Sigma_{g, 1}$. For each mapping class $\varphi \in \mathcal{M}_{g, 1}$, we have a homology

Received 19th March, 2007

The author would like to express his gratitude to Professor Shigeyuki Morita for his encouragement and helpful suggestions. The author also would like to thank the referee for his-or-her helpful comments. This research was supported by JSPS Research Fellowships for Young Scientists.

Copyright Clearance Centre, Inc. Serial-fee code: 0004-9727/07 \$A2.00+0.00. 
cylinder $\left(\Sigma_{g, 1} \times I\right.$, id $\left.\times 1, \varphi \times 0\right)$. In fact, this construction gives an injective monoid homomorphism $\mathcal{M}_{g, 1} \rightarrow \mathcal{C}_{g, 1}$. We can also construct homology cylinders by using homology 3 -spheres and pure string links (see $[3,7]$ ). From these facts, we can expect that $\mathcal{C}_{g, 1}$ will play an important role in the classification of 3 -manifolds especially by using its monoid structure.

To study the structure of $\mathcal{C}_{g, 1}$, we defined its Magnus representation

$$
r_{k}: \mathcal{C}_{g, 1} \rightarrow G L\left(2 g, \mathcal{K}_{N_{k}}\right) \quad(k=2,3, \ldots)
$$

in $[13,14]$, where $\mathcal{K}_{N_{k}}$ is a certain skew field. This representation extends the Magnus representation for $\mathcal{M}_{g, 1}$ defined by Morita [8], as the Gassner representation for pure string links due to Le Dimet [6] and Kirk, Livingston and Wang [5] does that for the pure braid group. See Birman's book [1] for generalities of the ordinary Magnus representation.

In this paper, we shall show that the Magnus representation of $\mathcal{C}_{g, 1}$ satisfies a certain equality which can be regarded as a non-commutative symplectic relation (Theorem 2.4). This generalises not only the result of Morita [8] and Suzuki [16] that the same equality holds for the Magnus representation of $\mathcal{M}_{g, 1}$, but also Kirk, Livingston and Wang's result [5] that the (reduced) Gassner representation for pure string links satisfies some unitary relation. In fact, our proof derives an explicit equality describing the unitarity of the Gassner representation, while their result is given in an implicit form.

Note that the statement of Theorem 2.4 has been already announced in [14], where we observe that the condition obtained from our theorem for a matrix to be written as the Magnus matrix of a homology cylinder is a strong one by considering a relationship to the theory of higher-order Alexander invariants.

\section{THE MAGNUS REPRESENTATION FOR HOMOLOGY CYLINDERS}

We take a base point $p$ on $\partial \Sigma_{g, 1}$, and take $2 g$ loops $\gamma_{1}, \ldots, \gamma_{2 g}$ of $\Sigma_{g, 1}$ as shown in Figure 1. We consider them to be an embedded bouquet $R_{2 g}$ of $2 g$ circles tied at $p \in \partial \Sigma_{g, 1}$. Then $R_{2 g}$ and the boundary loop $\zeta$ of $\Sigma_{g, 1}$ together with one 2-cell make up a standard cell decomposition of $\Sigma_{g, 1}$. The fundamental group $\pi_{1} \Sigma_{g, 1}$ of $\Sigma_{g, 1}$ is the free group $F_{2 g}$ of rank $2 g$ generated by $\gamma_{1}, \ldots, \gamma_{2 g}$, where $\zeta=\prod_{i=1}^{g}\left[\gamma_{i}, \gamma_{g+i}\right]$.

For a group $G$, we denote by $N_{k}(G):=G /\left(\Gamma^{k} G\right)$ the $k$-th nilpotent quotient of $G$, where we define $\Gamma^{1} G:=G$ and $\Gamma^{l} G:=\left[\Gamma^{l-1} G, G\right]$ for $l \geqslant 2$. For simplicity, we write $N_{k}(X)$ for $N_{k}\left(\pi_{1} X\right)$ where $X$ is a connected topological space, and write $N_{k}$ for $N_{k}\left(F_{2 g}\right)=N_{k}\left(\Sigma_{g, 1}\right)$.

Let $\left(M, i_{+}, i_{-}\right)$be a homology cylinder. By definition, $i_{+}, i_{-}: \pi_{1} \Sigma_{g, 1} \rightarrow \pi_{1} M$ are both 2-connected, namely they induce isomorphisms on $H_{1}$ and epimorphisms on $\mathrm{H}_{2}$. Then, by Stallings' theorem [15], $i_{+}, i_{-}: N_{k} \stackrel{\cong}{\rightarrow} N_{k}(M)$ are isomorphisms for each $k \geqslant 2$. 


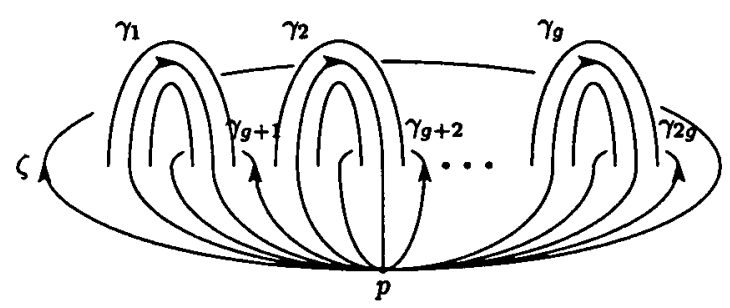

Figure 1: A standard cell decomposition of $\Sigma_{g, 1}$

Using them, we obtain a monoid homomorphism

$$
\sigma_{k}: \mathcal{C}_{g, 1} \rightarrow \text { Aut } N_{k} \quad\left(\left(M, i_{+}, i_{-}\right) \mapsto\left(i_{+}\right)^{-1} \circ i_{-}\right) .
$$

\subsection{Definition of the MAgNUS REPRESENTATION FOR hOMOLOGY CYLINDERS.}

We first summarise our notation. For a matrix $A$ with entries in a ring $R$, and a ring homomorphism $\varphi: R \rightarrow R^{\prime}$, we denote by ${ }^{\varphi} A$ the matrix obtained from $A$ by applying $\varphi$ to each entry. $A^{T}$ denotes the transpose of $A$. When $R=\mathbb{Z} G$ for a group $G$ or its right field of fractions (if it exists), we denote by $\bar{A}$ the matrix obtained from $A$ by applying the involution induced from $\left(x \mapsto x^{-1}, x \in G\right)$ to each entry. For a module $M$, we write $M^{n}$ for the module of column vectors with $n$ entries.

For a finite cell complex $X$, we denote by $\tilde{X}$ its universal covering. We take a base point $p$ of $X$ and a lift $\tilde{p}$ of $p$ as a base point of $\widetilde{X} . \pi:=\pi_{1}(X, p)$ acts on $\widetilde{X}$ from the right through its deck transformation group. Namely, the lift of $\gamma \in \pi$ starting from $\tilde{p}$ reaches $\tilde{p} \gamma^{-1}$. We regard the $\mathbb{Z} \pi$-cellular chain complex $C_{*}(\tilde{X})$ of $\tilde{X}$ as a collection of free right $\mathbb{Z} \pi$-modules consisting of column vectors together with differentials given by left multiplications of matrices. For each $\mathbb{Z} \pi$-bimodule $A$, the twisted chain complex $C_{*}(X ; A)$ is given by the tensor product of the right $\mathbb{Z} \pi$-module $C_{*}(\tilde{X})$ and the left $\mathbb{Z} \pi$-module $A$, so that $C_{*}(X ; A)$ and $H_{*}(X ; A)$ are right $\mathbb{Z} \pi$-modules.

Hereafter, we fix an integer $k \geqslant 2$. The following construction is based on KirkLivingston-Wang's work of the Gassner representation for string links in [5].

Let $\left(M, i_{+}, i_{-}\right)$be a homology cylinder. $p \in \partial \Sigma_{g, 1}$ is a common base point of $\Sigma_{g, 1}$ and $M$. By Stallings' theorem, $N_{k}$ and $N_{k}(M)$ are isomorphic. Since $N_{k}$ is a finitely generated torsion-free nilpotent group for each $k \geqslant 2$, we can embed $\mathbf{Z} N_{k}$ into the right field of fractions

$$
\mathcal{K}_{N_{k}}:=\mathbb{Z} N_{k}\left(\mathbb{Z} N_{k}-\{0\}\right)^{-1}
$$

(see $[2,11]$ for details). Similarly, we have

$$
\mathbf{Z} N_{k}(M) \hookrightarrow \mathcal{K}_{N_{k}(M)}:=\mathbf{Z} N_{k}(M)\left(\mathbf{Z} N_{k}(M)-\{0\}\right)^{-1} .
$$

By a standard argument (see for instance [5, Proposition 2.1], [13, Lemma 5.11]), 
we can show that

$$
i_{+}, i_{-}: H_{*}\left(\Sigma_{g, 1}, p ; i_{ \pm}^{*} \mathcal{K}_{N_{k}(M)}\right) \rightarrow H_{*}\left(M, p ; \mathcal{K}_{N_{k}(M)}\right)
$$

are isomorphisms as right $\mathcal{K}_{N_{k}(M)}$-vector spaces. Since $R_{2 g} \subset \Sigma_{g, 1}$ is a deformation retract, we have

$$
H_{1}\left(\Sigma_{g, 1}, p ; i_{ \pm}^{*} \mathcal{K}_{N_{k}(M)}\right) \cong H_{1}\left(R_{2 g}, p ; i_{ \pm}^{*} \mathcal{K}_{N_{k}(M)}\right)=C_{1}\left(\widetilde{R_{2 g}}\right) \bigotimes_{\mathrm{Z}_{1} R_{2 g}} i_{ \pm}^{*} \mathcal{K}_{N_{k}(M)} \cong \mathcal{K}_{N_{k}(M)}^{2 g}
$$

with a basis

$$
\left\{\tilde{\gamma_{1}} \otimes 1, \ldots, \widetilde{\gamma_{2 g}} \otimes 1\right\} \subset C_{1}\left(\widetilde{R_{2 g}}\right) \bigotimes_{\mathrm{z}_{1} R_{2 g}} i_{ \pm}^{*} \mathcal{K}_{N_{k}(M)}
$$

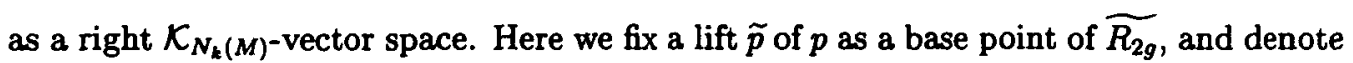
by $\tilde{\gamma}_{i}$ the lift of the oriented edge $\gamma_{i}$ starting from $\tilde{p}$ and ending at $\tilde{p} \gamma_{i}^{-1}$.

Definition 2.1: For each $M=\left(M, i_{+}, i_{-}\right) \in \mathcal{C}_{g, 1}$, we write $r_{k}^{\prime}(M) \in G L\left(2 g, \mathcal{K}_{N_{k}(M)}\right)$ for the representation matrix of the right $\mathcal{K}_{N_{k}(M)}$-isomorphism

$$
\begin{aligned}
\mathcal{K}_{N_{k}(M)}^{2 g} \cong H_{1}\left(\Sigma_{g, 1}, p ; i_{-}^{*} \mathcal{K}_{N_{k}(M)}\right) & \stackrel{\cong}{\underset{i_{-}}{\rightrightarrows}} H_{1}\left(M, p ; \mathcal{K}_{N_{k}(M)}\right) \\
& \underset{i_{+}^{-1}}{\cong} H_{1}\left(\Sigma_{g, 1}, p ; i_{+}^{*} \mathcal{K}_{N_{k}(M)}\right) \cong \mathcal{K}_{N_{k}(M)}^{2 g}
\end{aligned}
$$

(2) The Magnus representation for $\mathcal{C}_{g, 1}$ is the map $r_{k}: \mathcal{C}_{g, 1} \rightarrow G L\left(2 g, \mathcal{K}_{N_{k}}\right)$ which assigns to $M=\left(M, i_{+}, i_{-}\right) \in \mathcal{C}_{g, 1}$ the matrix ${ }^{i-1} r_{k}^{\prime}(M)$.

EXAMPLE 2.2. For $\varphi \in \mathcal{M}_{g, 1} \hookrightarrow$ Aut $F_{2 g}$, we can easily check that

$$
r_{k}\left(\left(\Sigma_{g, 1} \times I, \text { id } \times 1, \varphi \times 0\right)\right)=\overline{\overline{\rho_{k}}\left(\frac{\partial \varphi\left(\gamma_{j}\right)}{\partial \gamma_{i}}\right)_{i, j}}
$$

where $\rho_{k}: \mathbb{Z} F_{2 g} \rightarrow \mathbb{Z} N_{k} \subset \mathcal{K}_{N_{k}}$ is the natural homomorphism and $\partial / \partial \gamma_{i}$ are free differentials. From this, we see that $r_{k}$ extends the Magnus representation for $\mathcal{M}_{g, 1}$ (see $[8,16])$.

While we call $r_{k}$ the Magnus "representation", it is actually a crossed homomorphism. Namely, we have that following.

THEOREM 2.3. ([14]) For $M_{1}, M_{2} \in \mathcal{C}_{g, 1}$, we have

$$
r_{k}\left(M_{1} \cdot M_{2}\right)=r_{k}\left(M_{1}\right) \cdot{ }^{\sigma}\left(M_{1}\right) r_{k}\left(M_{2}\right)
$$

2.2. MAIN THEOREM. The following is the main theorem of this paper, which implies the symplecticity (in a twisted sense) of the Magnus reprentation for $\mathcal{C}_{g, 1}$. 
THEOREM 2.4. For any homology cylinder $M$, we have the equality

$$
\overline{r_{k}(M)^{T}} \tilde{J} r_{k}(M)={ }^{\sigma_{k}(M)} \tilde{J}
$$

where $\tilde{J}=\left(\begin{array}{ll}J_{1} & J_{2} \\ J_{3} & J_{4}\end{array}\right) \in G L\left(2 g, \mathbf{Z} N_{k}\right)$ is defined by

$$
\begin{aligned}
& J_{1}=\left(\begin{array}{ccccc}
1-\gamma_{1} & & & & 0 \\
\left(1-\gamma_{2}\right)\left(1-\gamma_{1}^{-1}\right) & 1-\gamma_{2} & & & 0 \\
\left(1-\gamma_{3}\right)\left(1-\gamma_{1}^{-1}\right) & \left(1-\gamma_{3}\right)\left(1-\gamma_{2}^{-1}\right) & 1-\gamma_{3} & & \\
\vdots & \vdots & & \ddots & \\
\left(1-\gamma_{g}\right)\left(1-\gamma_{1}^{-1}\right) & \left(1-\gamma_{g}\right)\left(1-\gamma_{2}^{-1}\right) & \cdots & & 1-\gamma_{g}
\end{array}\right) \\
& J_{2}=\left(\begin{array}{ccccc}
\gamma_{1} \gamma_{g+1}^{-1} & & & & 0 \\
\left(1-\gamma_{2}\right)\left(1-\gamma_{g+1}^{-1}\right) & \gamma_{2} \gamma_{g+2}^{-1} & & & 0 \\
\left(1-\gamma_{3}\right)\left(1-\gamma_{g+1}^{-1}\right) & \left(1-\gamma_{3}\right)\left(1-\gamma_{g+2}^{-1}\right) & \gamma_{3} \gamma_{g+3}^{-1} & & \\
\vdots & \vdots & & \ddots & \\
\left(1-\gamma_{g}\right)\left(1-\gamma_{g+1}^{-1}\right) & \left(1-\gamma_{g}\right)\left(1-\gamma_{g+2}^{-1}\right) & \cdots & & \gamma_{g} \gamma_{2 g}^{-1}
\end{array}\right) \text {, } \\
& J_{3}=\left(\begin{array}{ccccc}
1-\gamma_{1}^{-1}-\gamma_{g+1} & & & & 0 \\
\left(1-\gamma_{g+2}\right)\left(1-\gamma_{1}^{-1}\right) & 1-\gamma_{2}^{-1}-\gamma_{g+2} & & & \\
\left(1-\gamma_{g+3}\right)\left(1-\gamma_{1}^{-1}\right) & \left(1-\gamma_{g+3}\right)\left(1-\gamma_{2}^{-1}\right) & 1-\gamma_{3}^{-1}-\gamma_{g+3} & & \\
\vdots & \vdots & & \ddots & \\
\left(1-\gamma_{2 g}\right)\left(1-\gamma_{1}^{-1}\right) & \left(1-\gamma_{2 g}\right)\left(1-\gamma_{2}^{-1}\right) & \ldots & & 1-\gamma_{g}^{-1}-\gamma_{2 g}
\end{array}\right) \text {, } \\
& J_{4}=\left(\begin{array}{ccccc}
1-\gamma_{g+1}^{-1} & & & & 0 \\
\left(1-\gamma_{g+2}\right)\left(1-\gamma_{g+1}^{-1}\right) & 1-\gamma_{g+2}^{-1} & & \\
\left(1-\gamma_{g+3}\right)\left(1-\gamma_{g+1}^{-1}\right) & \left(1-\gamma_{g+3}\right)\left(1-\gamma_{g+2}^{-1}\right) & 1-\gamma_{g+3}^{-1} & & \\
\vdots & \vdots & & \ddots & \\
\left(1-\gamma_{2 g}\right)\left(1-\gamma_{g+1}^{-1}\right) & \left(1-\gamma_{2 g}\right)\left(1-\gamma_{g+2}^{-1}\right) & \ldots & & 1-\gamma_{2 g}^{-1}
\end{array}\right) \text {. }
\end{aligned}
$$

Note that the matrix $\widetilde{J}$ appeared in Papakyriakopoulos' paper [10], and that it is mapped to the ordinary symplectic matrix by the augmentation map $\mathbb{Z} N_{k} \rightarrow \mathbb{Z}$.

Theorem 2.4 is a generalisation of the result of Morita [8] and Suzuki [16] that the Magnus representation for $\mathcal{M}_{g, 1}$ has the same symplecticity as above. Morita used a finite generating system of $\mathcal{M}_{g, 1}$ and showed that the equality holds for each element of the system. On the other hand, Suzuki used twisted homology groups of $\Sigma_{g, 1}$ to show that, without using any generating system, every element of $\mathcal{M}_{g, 1}$ preserves a related intersection pairing (called higher intersection number), which implies the equality. In our case, we shall give a proof which is closer to the latter, since no generating system is known for $\mathcal{C}_{g, 1}$ at present. Also, it is not known whether $\mathcal{C}_{g, 1}$ is finitely generated or not. 


\section{ProOF OF THEOREM 2.4}

Let $\left(M, i_{+}, i_{-}\right) \in \mathcal{C}_{g, 1}$. The proof of Theorem 2.4 is divided into the following three steps.

STEP 1. Triangulate $\Sigma_{g, 1}$ by taking a refinement of the cell decomposition in Figure 1. We decompose $\partial \Sigma_{g, 1}$ into two segments $A$ and $B$ such that $A \cup B=\partial \Sigma_{g, 1}, \partial A=\partial B$ $=A \cap B=\{p, q\}$ as in Figure 2.

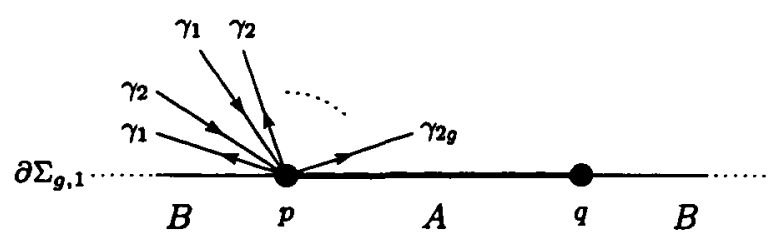

Figure 2: Decomposing $\partial \Sigma_{g, 1}$ into $A$ and $B$

Then we have isomorphisms

$$
\begin{aligned}
& H_{1}\left(\Sigma_{g, 1}, p ; \mathcal{K}_{N_{k}}\right) \cong H_{1}\left(\Sigma_{g, 1}, A ; \mathcal{K}_{N_{k}}\right), \\
& H_{1}\left(\Sigma_{g, 1}, p ; \mathcal{K}_{N_{k}}\right) \cong H_{1}\left(\Sigma_{g, 1}, A ; \mathcal{K}_{N_{k}}\right) \cong H_{1}\left(\Sigma_{g, 1}, q ; \mathcal{K}_{N_{k}}\right) \cong H_{1}\left(\Sigma_{g, 1}, B ; \mathcal{K}_{N_{k}}\right) .
\end{aligned}
$$

Using them, we obtain a pairing

$$
\begin{aligned}
\langle,\rangle_{g, 1}: H_{1}\left(\Sigma_{g, 1}, p ; \mathcal{K}_{N_{k}}\right) \otimes H_{1}\left(\Sigma_{g, 1}, p ; \mathcal{K}_{N_{k}}\right) & \stackrel{\cong}{\rightarrow} H_{1}\left(\Sigma_{g, 1}, A ; \mathcal{K}_{N_{k}}\right) \otimes H_{1}\left(\Sigma_{g, 1}, B ; \mathcal{K}_{N_{k}}\right) \\
& \rightarrow \mathcal{K}_{N_{k}}
\end{aligned}
$$

where the second map is the intersection pairing given, for instance, in [12, Definition 4.66]. More precisely, we need to generalise the pairing in the book to the case of pairs of manifolds and enlarge its coefficients from $Z N_{k}$ to $\mathcal{K}_{N_{k}}$. In our notation, the formula for the corresponding chain-level intersection

$$
\langle,\rangle_{\Sigma_{g, 1}}:\left(C_{1}\left(\widetilde{\Sigma_{g, 1}}, \bar{A}\right) \bigotimes_{\mathbf{z}_{1} \Sigma_{g, 1}} \mathcal{K}_{N_{k}}\right) \otimes\left(D_{1}\left(\widetilde{\Sigma_{g, 1}}, \bar{B}\right) \bigotimes_{\mathbf{z}_{1} \Sigma_{g, 1}} \mathcal{K}_{N_{k}}\right) \rightarrow \mathcal{K}_{N_{k}}
$$

of the simplicial 1-chains

$$
C_{1}\left(\Sigma_{g, 1}, A ; \mathcal{K}_{N_{k}}\right)=C_{1}\left(\widetilde{\Sigma_{g, 1}}, \bar{A}\right) \underset{z_{\pi_{1} \Sigma_{g, 2}}}{\bigotimes} \mathcal{K}_{N_{k}}
$$

and the dual cellular 1-chains

$$
D_{1}\left(\Sigma_{g, 1}, B ; \mathcal{K}_{N_{k}}\right)=D_{1}\left(\widetilde{\Sigma_{g, 1}}, \bar{B}\right) \bigotimes_{\pi_{1} \Sigma_{g, 1}} \mathcal{K}_{N_{k}}
$$


(see [9, Chapter 8]), where $\bar{A}$ and $\bar{B}$ denote the inverse images of $A$ and $B$ in $\widetilde{\Sigma_{g, 1}}$, is given as follows: For $u \in C_{1}\left(\widetilde{\Sigma_{g, 1}}, \bar{A}\right), v \in D_{1}\left(\widetilde{\Sigma_{g, 1}}, \bar{B}\right)$ and $r, s \in \mathcal{K}_{N_{k}}$,

$$
\langle u \otimes r, v \otimes s\rangle_{\Sigma_{g, 1}}=\sum_{\gamma \in \pi_{1} \Sigma_{g, 1}}(u \gamma, v) \bar{r} \gamma s,
$$

where $(u \gamma, v) \in \mathbb{Z}$ is the usual intersection number of $u \gamma$ and $v$ on $\widetilde{\Sigma_{g, 1}}$.

By definition, $\langle u f, v\rangle_{\Sigma_{g, 1}}=\bar{f}\langle u, v\rangle_{\Sigma_{g, 1}}$ and $\langle u, v f\rangle_{\Sigma_{g, 1}}=\langle u, v\rangle_{\Sigma_{g, 1}} f$ hold for any $f \in \mathcal{K}_{N_{k}}$ and $u, v \in H_{1}\left(\Sigma_{g, 1}, p ; \mathcal{K}_{N_{k}}\right)$. Note that this pairing generalises Suzuki's higher intersection number in [16].

STEP 2. We glue two copies of $\Sigma_{g, 1}$ along $A$ to construct a surface $S$ with the natural inclusions $i_{+}^{\prime}, i_{-}^{\prime}: \Sigma_{g, 1} \hookrightarrow S$. We orient $S$ so that $i_{+}^{\prime}$ is orientation-preserving. $S$ is a surface of genus $2 g$ with the boundary $i_{+}^{\prime}(B) \cup i_{-}^{\prime}(B)$. Capping $S$ by a disk $D$, we obtain a closed surface of genus $2 g$, which is naturally identified with $\partial M$ so that $i_{+}$and $i_{-}$are homotopic to $i_{+}^{\prime}$ and $i_{-}^{\prime}$ respectively. We can extend the triangulation of $\Sigma_{g, 1}$ to those of $\partial M$ and $M$.

Similarly to the above, we define a pairing

$$
\langle,\rangle_{S}: H_{1}\left(S, p ; \mathcal{K}_{N_{k}(M)}\right) \otimes H_{1}\left(S, p ; \mathcal{K}_{N_{k}(M)}\right) \rightarrow \mathcal{K}_{N_{k}(M)}
$$

by using

$$
\begin{aligned}
& H_{1}\left(S, p ; \mathcal{K}_{N_{k}(M)}\right) \cong H_{1}\left(S, i_{+}^{\prime}(B) ; \mathcal{K}_{N_{k}(M)}\right) \\
& H_{1}\left(S, p ; \mathcal{K}_{N_{k}(M)}\right) \cong H_{1}\left(S, A ; \mathcal{K}_{N_{k}(M)} \cong H_{1}\left(S, q ; \mathcal{K}_{N_{k}(M)} \cong H_{1}\left(S, i_{-}^{\prime}(B) ; \mathcal{K}_{N_{k}(M)}\right)\right.\right.
\end{aligned}
$$

Then we can check that

$$
{ }^{i_{+}}\langle u, v\rangle_{\Sigma_{g, 1}}=\left\langle i_{+}^{\prime}(u), i_{+}^{\prime}(v)\right\rangle_{S}, \quad{ }^{i}\langle u, v\rangle_{\Sigma_{g, 1}}=-\left\langle i_{-}^{\prime}(u), i_{-}^{\prime}(v)\right\rangle_{S} \quad \in \mathcal{K}_{N_{k}(M)}
$$

by an argument similar to that in Step 3. We also have a pairing

$$
\langle,\rangle_{M}: H_{1}\left(M, D ; \mathcal{K}_{N_{k}(M)}\right) \otimes H_{2}\left(M, S ; \mathcal{K}_{N_{k}(M)}\right) \rightarrow \mathcal{K}_{N_{k}(M)} .
$$

To see the relationship between $\langle,\rangle_{S}$ and $\langle,\rangle_{M}$, we recall that the diagram

$$
\begin{array}{ccc}
H^{1}\left(M, D ; \mathcal{K}_{N_{k}(M)}\right) & \stackrel{i^{*}}{\longrightarrow} H^{1}\left(S, i_{+}^{\prime}(B) ; \mathcal{K}_{N_{k}(M)}\right) \\
\lfloor n[M, \partial M] & & \lfloor n[S, \partial S] \\
H_{2}\left(M, S ; \mathcal{K}_{N_{k}(M)}\right) & \stackrel{\theta}{\longrightarrow} & H_{1}\left(S, i_{-}^{\prime}(B) ; \mathcal{K}_{N_{k}(M)}\right)
\end{array}
$$

commutes up to sign, where $i: S \hookrightarrow M$ is the inclusion, the lower horizontal arrow is obtained from the homology exact sequence of the triplet $\left(M, S, i_{-}^{\prime}(B)\right)$, and we write $[M, \partial M] \in H_{3}(M, \partial M)$ and $[S, \partial S] \in H_{2}(S, \partial S)$ for the fundamental classes of $M$ and $S$ respectively. From this, we have

$$
\langle x, \partial y\rangle_{S}=-\langle i(x), y\rangle_{M}
$$


for any $x \in H_{1}\left(S, p ; \mathcal{K}_{N_{k}(M)}\right)$ and $y \in H_{2}\left(M, S ; \mathcal{K}_{N_{k}(M)}\right)$. Consequently,

$$
\begin{aligned}
i_{-}\langle u, v\rangle_{\Sigma_{g, 1}} & =-\left\langle i_{-}^{\prime}(u), i_{-}^{\prime}(v)\right\rangle_{S} \\
& =-\left\langle i_{-}^{\prime}(u), i_{-}^{\prime}(v)-i_{+}^{\prime}\left(r_{k}(M) v\right)\right\rangle_{S} \\
& =\left\langle i_{-}(u), X\right\rangle_{M} \\
& =\left\langle i_{+}\left(r_{k}(M) u\right), X\right\rangle_{M} \\
& =-\left\langle i_{+}^{\prime}\left(r_{k}(M) u\right), i_{-}^{\prime}(v)-i_{+}^{\prime}\left(r_{k}(M) v\right)\right\rangle_{S} \\
& =\left\langle i_{+}^{\prime}\left(r_{k}(M) u\right), i_{+}^{\prime}\left(r_{k}(M) v\right)\right\rangle_{S} \\
& ={ }^{i_{+}}\left\langle r_{k}(M), r_{k}(M)\right\rangle_{\Sigma_{g, 1}},
\end{aligned}
$$

where $\partial X=i_{-}^{\prime}(v)-i_{+}^{\prime}\left(r_{k}(M) v\right)$. By applying $i_{+}^{-1}$, we obtain the equality

$$
\left\langle r_{k}(M) u, r_{k}(M) v\right\rangle_{\Sigma_{g, 1}}={ }^{\sigma_{k}(M)}\langle u, v\rangle_{\Sigma_{g, 1}} .
$$

STEP 3. We shall describe the pairing $\langle,\rangle_{\Sigma_{g, 1}}$ explicitly by calculating the chain-level intersection of cycles representing the basis elements $\left\{\widetilde{\gamma_{1}} \otimes 1, \ldots, \widetilde{\gamma_{2 g}} \otimes 1\right\}$ of $H_{1}\left(\Sigma_{g, 1}, p ; \mathcal{K}_{N_{k}}\right)$. For that, we use Papakyriakopoulos' argument in [10]. In our notation, cycles intersect each other as in Figures 3 and 4 , where $\tilde{\gamma}_{i}^{*}$ is a path which represents the class corresponding to $\widetilde{\gamma}_{l} \otimes 1$ by the isomorphism $H_{1}\left(\Sigma_{g, 1}, p ; \mathcal{K}_{N_{k}}\right) \cong H_{1}\left(\Sigma_{g, 1}, B ; \mathcal{K}_{N_{k}}\right)$ mentioned in Step 1.

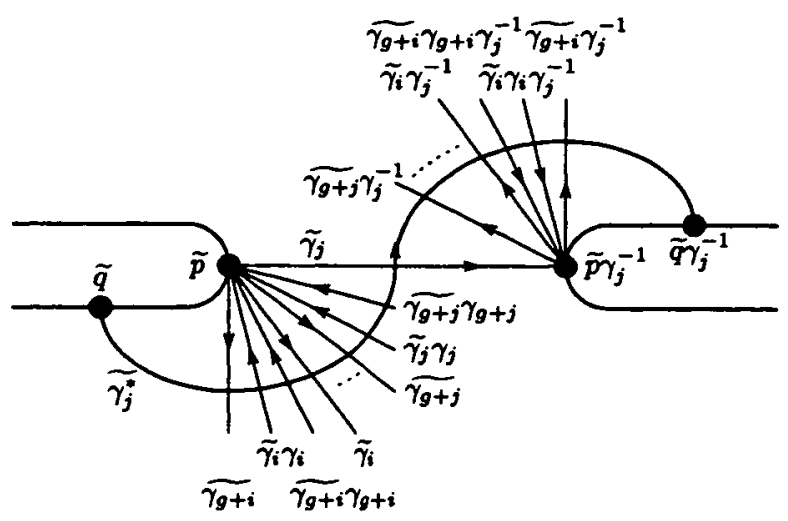

Figure 3: Intersections of lifts of edges (I)

From the figures, for example, we see that

$$
\left\langle\tilde{\gamma}_{i} \otimes 1, \tilde{\gamma}_{j} \otimes 1\right\rangle_{\Sigma_{g, 1}}=1-\gamma_{i}-\gamma_{j}^{-1}+\gamma_{i} \gamma_{j}^{-1}=\left(1-\gamma_{i}\right)\left(1-\gamma_{j}^{-1}\right)
$$

for $1 \leqslant j<i \leqslant g$. By computing all cases, we obtain the matrix $\widetilde{J}$. 


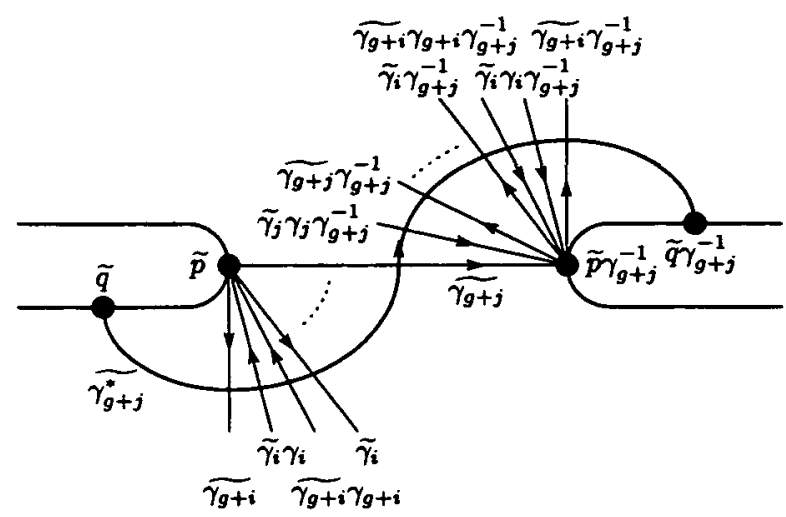

Figure 4: Intersections of lifts of edges (II)

REMARK 3.1. Our equality in Theorem 2.4 does depend on the choices involved in the proof. For example, by changing the generating system of $\pi_{1} \Sigma_{g, 1}$ and the rules for defining intersection pairings, we obtain other matrices than $\widetilde{J}$ representing the symplecticity of the Magnus representation.

\section{ThE GASSNER REPRESENTATION FOR STRING LINKS}

Let $D^{2}$ be a 2-dimensional disk. Given $n \geqslant 1$, we fix $n$ points $p_{1}, \ldots, p_{n}$ in the interior of $D^{2}$. A string link of $n$ strands is a smooth embedding of the disjoint union of ordered $n$ unit intervals into $D^{2} \times I$ sending 0 and 1 of $i$-th interval to $p_{i} \times\{0\}$ and $p_{\tau(i)} \times\{1\}$, where $\tau$ is an element of the symmetric group of degree $n$. The set $\mathcal{S} \mathcal{L}_{n}$ of isotopy classes of string links of $n$ strands has a natural monoid structure. A string link is said to be pure if $\tau=\mathrm{id}$, and we denote by $\mathcal{P S \mathcal { L } _ { n }}$ the submonoid of $\mathcal{S} \mathcal{L}_{n}$ consisting of all pure string links.

Let $D_{n}:=D^{2}-\left\{p_{1}, \ldots, p_{n}\right\} . \pi_{1} D_{n}$ is the free group generated by $n$ loops $\delta_{1}, \ldots, \delta_{n}$ as in Figure 5. In this section, we put $N_{k}:=N_{k}\left(\pi_{1} D_{n}\right)$. We write $i_{0}$ and $i_{1}$ for the natural embeddings of $D_{n}$ into $D^{2} \times\{0\}$ and $D^{2} \times\{1\}$ respectively. It is easily checked that $i_{0}, i_{1}: \pi_{1} D_{n} \rightarrow \pi_{1}\left(D^{2} \times I-N(L)\right)$ are 2-connected for each string link $L$, where $N(L)$ denotes a tubular neighbourhood of (the image of) $L$. Hence a construction similar to that in Section 2 gives a monoid homomorphism

$$
\sigma_{G, k}: S \mathcal{L}_{n} \rightarrow \text { Aut } N_{k} \quad\left(L \mapsto\left(i_{1}\right)^{-1} \circ i_{0}\right)
$$

for each $k \geqslant 2$. Note that $\operatorname{Ker} \sigma_{G, 2}=\mathcal{P} \mathcal{S} \mathcal{L}_{n}$. By using $H_{1}\left(D_{n}, p ; \mathcal{K}_{N_{k}}\right)$, we also have a crossed homomorphism

$$
r_{G, k}: \mathcal{S} \mathcal{L}_{n} \rightarrow G L\left(n, \mathcal{K}_{N_{k}}\right)
$$




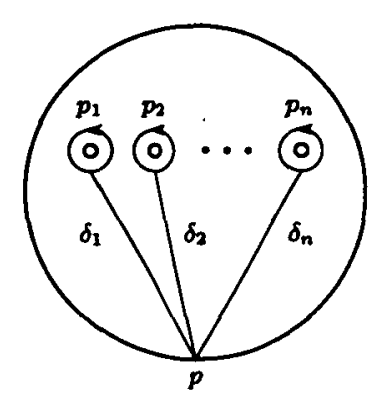

Figure 5: A generating system of $\pi_{1} D_{n}$

called the Gassner representation for string links. We refer to $[6,5]$ for details. (In these

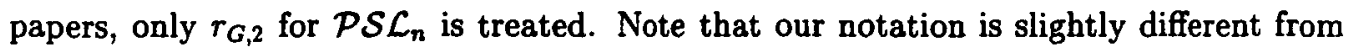
theirs.)

THEOREM 4.1. For any string link $L$ of $n$ strands, we have the equality

$$
\overline{r_{G, k}(L)^{T}} J_{4}^{\prime} r_{G, k}(L)={ }^{\sigma, k}(L) J_{4}^{\prime},
$$

where

$$
J_{4}^{\prime}=\left(\begin{array}{ccccc}
1-\delta_{1}^{-1} & & & & 0 \\
\left(1-\delta_{2}\right)\left(1-\delta_{1}^{-1}\right) & 1-\delta_{2}^{-1} & & & 0 \\
\left(1-\delta_{3}\right)\left(1-\delta_{1}^{-1}\right) & \left(1-\delta_{3}\right)\left(1-\delta_{2}^{-1}\right) & 1-\delta_{3}^{-1} & & \\
\vdots & \vdots & & \ddots & \\
\left(1-\delta_{n}\right)\left(1-\delta_{1}^{-1}\right) & \left(1-\delta_{n}\right)\left(1-\delta_{2}^{-1}\right) & \ldots & & 1-\delta_{n}^{-1}
\end{array}\right)
$$

Proof: Since the proof goes parallel to that of Theorem 2.4, we omit the details. Instead, we here explain that this result is obtained as a corollary of Theorem 2.4 when

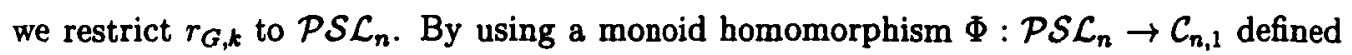
by Levine [7], we showed in [14] that

$$
r_{k}(\Phi(L))=\left(\begin{array}{cc}
* & 0_{n} \\
* & r_{G, k}(L)
\end{array}\right)
$$

holds for each pure string link $L$, where we identify $\gamma_{n+i} \in \pi_{1} \Sigma_{n, 1}$ with $\delta_{i} \in \pi_{1} D_{n}$ for $i=1, \ldots, n$. Then our claim follows from Theorem 2.4 by seeing the lower right part. $\square$

\section{REFERENCES}

[1] J. Birman, Braids, links and mapping class groups, Ann. of Math. Stud. 82 (Princeton Univ. Press, Princeton, N.J., 1974).

[2] P.M. Cohn, Skew fields; Theory of general division rings, Encyclopedia Math. Appl. (Cambridge Univ. Press, London, 1995). 
[3] S. Garoufalidis and J. Levine, 'Tree-level invariants of three-manifolds, Massey products and the Johnson homomorphism', in Graphs and patterns in mathematics and theoretical physics, Proc. Sympos. Pure Math. 73, 2005, pp. 173-205.

[4] K. Habiro, 'Claspers and finite type invariants of links', Geom. Topol. 4 (2000), 1-83.

[5] P. Kirk, C. Livingston and Z. Wang, 'The Gassner representation for string links', Commun. Contemp. Math. 1 (2001), 87-136.

[6] J.Y. Le Dimet, 'Enlacements d'intervalles et représentation de Gassner', Comment. Math. Helv. 67 (1992), 306-315.

[7] J. Levine, 'Homology cylinders: an enlargement of the mapping class group', Algebr. Geom. Topol. 1 (2001), 243-270.

[8] S. Morita, 'Abelian quotients of subgroups of the mapping class group of surfaces', Duke Math. J. 70 (1993), 699-726.

[9] J.R. Munkres, Elements of algebraic topology (Addison-Wesley Publishing Company, Menlo Park, CA, 1984).

[10] C.D. Papakyriakopoulos, 'Planar regular coverings of orientable closed surfaces', in Ann. of Math. Stud. 84 (Princeton Univ. Presss, Princeton, N.J., 1975), pp. 261-292.

[11] D. Passman, The algebraic structure of group rings (John Wiley and Sons, New York, London, Sydney, 1977).

[12] A. Ranicki, Algebraic and geometric surgery (Oxford University Press, Oxford, 2002).

[13] T. Sakasai, 'Homology cylinders and the acyclic closure of a free group', Algeb. Geom. Topol. 6 (2006), 603-631.

[14] T. Sakasai, 'The Magnus representation and higher-order Alexander invariants for homology cobordisms of surfaces', (preprint, arXiv:math.GT/0507266).

[15] J. Stallings, 'Homology and central series of groups', J. Algebra 2 (1965), 170-181.

[16] M. Suzuki, 'Geometric interpretation of the Magnus representation of the mapping class group', Kobe J. Math. 22 (2005), 39-47.

Graduate School of Mathematical Sciences

The University of Tokyo

3-8-1 Komaba, Meguro-ku

Tokyo 153-8914

Japan

e-mail: sakasai@ms.u-tokyo.ac.jp 